\title{
Relay-Assisted Transmission with Fairness Constraint for Cellular Networks
}

\author{
Erwu Liu, Member, IEEE, Qinqing Zhang, Senior Member, IEEE, and Kin K. Leung, Fellow, IEEE
}

\begin{abstract}
We consider the problem of relay-assisted transmission for cellular networks. In the considered system, a source node together with $n$ relay nodes are selected in a proportionally fair (PF) manner to transmit to the base station (BS), which uses the maximal ratio combining (MRC) to combine the signals received from the source node in the first half slot and the $n$ relay nodes in the second half slot for successful reception. The proposed algorithm incorporates the $\mathrm{PF}$ criterion and cooperative diversity, and is called proportionally fair cooperation (PFC). Compared with the proportional fair scheduling (PFS) algorithm, PFC provides improved efficiency and fairness.

The ordinary differential equation (ODE) analysis used to study PFS cannot be used for PFC, otherwise one has to solve a large number of nonlinear and interrelated ODE equations which is time-prohibited. In this paper, we present a mathematical framework for the performance of PFC. The cornerstone of our framework is a realistic yet simple model that captures node cooperation, fading, and fair resource allocation-induced dependencies. We obtain analytical expressions for the throughput gain of PFC over traditional PFS without node cooperation. Compared with the highly time-consuming ordinary differential equation (ODE) analysis, our formulae are intuitive yet easy to evaluate numerically.

To our knowledge, it is the first time that a closed-form expression is obtained for the throughput of relay-assisted transmission in a cellular network with the PF constraint.
\end{abstract}

Index Terms-relay-assisted transmission, proportional fairness, cellular networks.

\section{INTRODUCTION}

$\mathbf{T}$ HIS paper proposes and analyzes a practical cooperative diversity scheme for cellular networks. The rationale for the extraordinary interest in exploiting cooperative techniques in wireless networks is very clear, as demonstrated by the high volume of publications in recent years [1]-[11]. In this direction, we consider a cooperative diversity scheme, i.e., relay-assisted transmission which requires minimum cooperation among nodes, for time-division-multiple-access (TDMA) cellular networks and focuses on the throughput performance.

Inspired by the opportunistic relaying method proposed by Bletsas [6] wherein one relay $r$ with the best source-relaydestination path is selected for relaying, this research considers the problem of how to select the set of relays with the "best" source-relayset-destination path for relaying. Specifically, in a TDMA cellular network with relay-assisted transmission, a

Erwu Liu was with the Department of Electrical and Electronic Engineering, Imperial College, London, UK. He is also with the School of Electronics and Information, Tongji University, Shanghai, CHINA, Email: erwu.liu@ieee.org

Qinqing Zhang is with the Department of Computer Science, Johns Hopkins University, USA, Email: qinqing.zhang@ jhuapl.edu

Kin K. Leung is with the Department of Electrical and Electronic Engineering, Imperial College, London, UK, Email: kkleung @ieee.org
Node $m$ together with an $n$-node relayset $\Phi(m \notin \Phi)$ are selected by the base station (BS) to transmit at next slot in a collaborative manner. There are totally $N\left(\begin{array}{c}N-1 \\ n\end{array}\right)(m, \Phi)$ pairs in an $N$-node cellular network. Once the "best" pair $(m, \Phi)$ is selected, Node $m$ will transmit and all nodes in $\Phi$ will function as decode-and-forward (DF) relays [4]. The BS will store the signal received from $m$ in the first half slot and use it together with the signals received from the $n$ relays in the second half slot for successful decoding. Without node cooperation, the BS will only decode using the signal received from $m$. With this scheme, better performance can be achieved as the BS will decode using the signals from $n+1$ branches.

Unlike network multiple-input multiple-output (MIMO) or distributed beamforming systems [12]-[15] where synchronization among distributed antennas is typically an issue, our method implements maximal ratio combining (MRC) for decoding, which does not require perfect synchronization among all branches and is typically seen in practical systems.

The performance of the considered system not only depends on node cooperation, it also depends on the metric used to select the $(m, \Phi)$ pair. In [16], a set of allocated rates is called proportionally fair (PF) if any other allocation results in non-positive aggregate change. Originally from Kelly's work [16], the proportionally fair scheduling (PFS) algorithm [17][21] has spurred the development of network utility maximization algorithms since 1997 [22]-[26], and is implemented in current 3G networks [27] as the most-cited NUM method. By assigning each user a rate that is inversely proportional to its anticipated resource consumption [17], PFS has shown excellent balance between throughput and fairness via multiuser diversity and game-theoretic equilibrium. In light of this, we apply the PF criterion in selecting the pair $(m, \Phi)$ for relayassisted transmission in cellular networks, and the scheduling algorithm is called proportionally fair cooperation (PFC).

Our objective is to analyze the performance of PFC. We would like to point out that the ordinary differential equation (ODE) analysis used to study PFS [17] can be extended to study PFC. In a recent research, Zhou and Fan etc. [28] have used the ODE analysis to study the throughput performance of cooperative proportional fair scheduling (CPF) for a multibase-station scenario, where multiple BSs cooperatively transmit to a user. In [17], Kushner and Whiting have proved that for an $N$-node cellular network, the long-term PFS throughputs of users can be obtained by solving $N$ ODE equations. Similarly, Zhou and Fan etc. [28] have proved that for an $M$-BS, $N$-node multi-base-station system, the longterm CPF throughputs of the users can be obtained by solving $M \times N$ ODE equations. 
While the ODE analysis is quite accurate, the problem is that it is highly time-consuming, especially because the ODE equations involved are nonlinear and interplay with each other in an intricate manner. For a network configuration of $N=20$ users, let say $n=2$ relays are used in relaying, then using the ODE analysis to study our PFC algorithm requires solving $N \times\left(\begin{array}{c}N-1 \\ n\end{array}\right)=3420$ non-linear ODEs. This is totally timeprohibited and thus new method is needed for the analysis of PFC. Towards this end, we derive a mathematical framework to quickly estimate the PFC throughput without using the highly time-consuming ODE analysis.

By simulation, it turns out that PFC considerably improves the system throughput while at the same time exhibits increased fairness compared with PFS, and the analytical model presented here is of closed-form and hence easy to evaluate. In our experiment, the analytical model provides highly accurate estimate of the simulated throughput for all users.

The paper is structured as follows. In Section II, we first describe relay-assisted transmission in cellular networks and the resulting problem formulations, we then propose the PFC algorithm that maximizes the overall utility of a cellular network with relay-assisted transmission. After that, analytical results of PFC are provided in Section III. Finally, Section IV conducts simulations to validate the theoretical model, and presents results to evaluate the throughput and fairness performance of PFC, followed by the conclusion in Section V.

\section{SySTEM MOdel}

We start with a TDMA cellular network without relays.

For an $N$-node TDMA cellular network, let's consider the problem where these $N$ nodes wish to transmit data to the $\mathrm{BS}$, and the rates of transmission are randomly varying due to channel fluctuations. Time is divided into small scheduling intervals called slots. The selection of a node to schedule is based on a balance between throughput and fairness. PFS [16] performs this by comparing the ratio of the achievable rate (i.e., capacity) for each node to its short-term average throughput tracked by an exponential moving average, which is defined as the preference metric. The node with the maximum preference metric will be selected for transmission at the next slot. This is described mathematically as follows. The end of slot $t$ is called time $t$. In next time slot $t+1$, the achievable data rate of Node $j$ will be $R_{j}[t+1]$. Its throughput up to time $t$ is denoted by $\mu_{j}[t]$, and the preference metric by $\mathbf{M}_{j}[t+1]=R_{j}[t+1] / \mu_{j}[t]$.

According to the PFS algorithm used in current $3 \mathrm{G}$ networks [27], Node $i=\arg \max _{j} \mathbf{M}_{j}[t+1]$ will be scheduled in next slot $t+1$. The throughput of Node $j$ is updated by

$$
\mu_{j}[t+1]=\left(1-\frac{1}{k}\right) \mu_{j}[t]+I_{j}[t+1] \times \frac{R_{j}[t+1]}{k} .
$$

where $k$ is the smooth factor (a positive typically $>50$ ), $I_{j}[t+1]$ is the indicator function of the event that Node $j$ is scheduled to transmit in slot $t+1$.

$$
I_{j}[t+1]= \begin{cases}1, & j \text { is scheduled in slot } t+1 \\ 0, & \text { else }\end{cases}
$$

It is known that the PFS algorithm above maximizes the overall utility $\sum_{i} U\left(\overline{\mu_{i}}\right)$ where $\overline{\mu_{i}}$ is the long-term average throughput, $U\left(\overline{\mu_{i}}\right)=\ln \left(\overline{\mu_{i}}\right)$ is the utility function defined for elastic flows [16].

\section{A. Relay-Assisted Transmission}

Now consider a TDMA cellular network with relays as shown in Fig. 1, where the source node $m$ transmits to the BS with the help of a set of $n$ relays $\Phi=\left\{r_{1}, r_{2}, \ldots, r_{n}\right\}$. Each node has a single antenna operating in half duplex mode, and can function as a DF relay as needed.

Unlike the BS in a traditional cellular network where only one node is selected for transmission at a time, the BS in the considered relay-assisted cellular network schedules a number of nodes $(m, \Phi)$ for transmission which occurs over two orthogonal half timeslots.

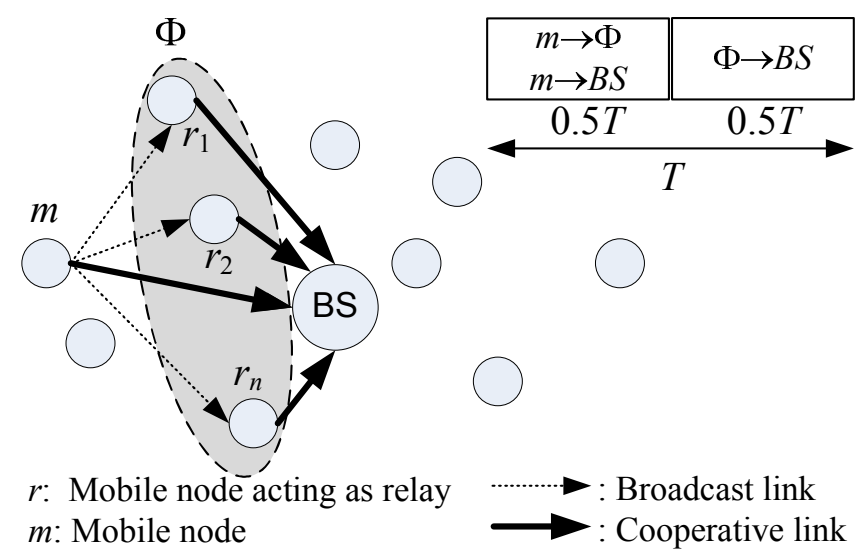

Fig. 1. Relay-assisted transmission in a cellular network

Let $\Psi$ denote the set of all nodes in the network, $\Theta$ the set of relayset $\Phi, \Theta=\{\Phi|| \Phi \mid=n\}$, and $\Omega=\{(m, \Phi) \mid m \in$ $\Psi, \Phi \in \Theta, m \notin \Phi\}$ the set of all possible pairs. For an $N$-node cellular network, there are totally $K=N\left(\begin{array}{c}N-1 \\ n\end{array}\right)(m, \Phi)$ pairs.

Refer to Fig. 1, a transmission over a broadcast link together with a transmission over the correspondingly cooperative link compose a two-phase, relay-assisted transmission. A relayassisted transmission is completed in two orthogonal half timeslots. Once a pair $(m, \Phi)$ is selected for relay-assisted transmission, Node $m$ will broadcast to both $\Phi$ and the BS with power $P_{m}$ during the first half timeslot. Note that $\Phi$ will decode while the BS will only store the signal received from $m$ in the first half timeslot. After successful decoding, relay node $r_{i} \in \Phi(i=1,2, \ldots, n)$ will transmit with power $P_{r_{i}}$ to the BS in the second half timeslot. The BS will then decode using the signals received from $\Phi$ in the second half timeslot and the stored signal received from $m$ in the first half timeslot.

Let $x_{m}$ be the symbol sent by $m, y_{B S, m}$ be the symbol received at the BS from $m$ during the first half timeslot, $y_{B S, r_{i}}$ be the symbol received at the BS from relay $r_{i}$ during the second half timeslot, and $y_{r_{i}, m}$ be the received symbol at $r_{i}$ from $m$ during the first half timeslot. The complex channel gains from $m$ to the BS, $m$ to $r_{i}$, and $r_{i}$ to the $\mathrm{BS}$ are denoted by $h_{m, B S}, h_{m, r_{i}}$, and $h_{r_{i}, B S}$ respectively. The channel gains 
are assumed to be identical in both half timeslots. The channel equations are

$$
\begin{gathered}
y_{B S, m}=\sqrt{P_{m}} h_{m, B S} x_{m}+n_{B S, m} . \\
y_{r_{i}, m}=\sqrt{P_{m}} h_{m, r_{i}} x_{m}+n_{r_{i}, m} . \\
y_{B S, r_{i}}=\sqrt{P_{r_{i}}} h_{r_{i}, B S} x_{m}+n_{B S, r_{i}} .
\end{gathered}
$$

where $n_{B S, m}, n_{r_{i}, m}$, and $n_{B S, r_{i}}$ are circularly symmetric complex Gaussian noise $C N\left(0, N_{0} W\right)$ at the $m \rightarrow B S$, $m \rightarrow r_{i}$, and $r_{i} \rightarrow B S$ links respectively, where $W$ is system bandwidth.

Unless otherwise specified, $W$ is normalized to $1 \mathrm{~Hz}$. We use $m \rightarrow \Phi,(m, \Phi) \rightarrow B S$ to denote broadcast and cooperative links. Let $R_{m, r_{i}}, R_{m, \Phi}^{B}$, and $R_{m, \Phi}^{C}$ be the achievable data rates of link $m \rightarrow r_{i}$, broadcast link $m \rightarrow \Phi$, and cooperative link $(m, \Phi) \rightarrow B S$. Successful decoding of $x_{m}$ at $r_{i}$ requires

$$
\begin{aligned}
R_{m, r_{i}} & =\log _{2}\left(1+\frac{P_{m}\left|h_{m, r_{i}}\right|^{2}}{N_{0} W}\right) \\
& =\log _{2}\left(1+S N R_{m, r_{i}}\right) .
\end{aligned}
$$

where $S N R_{m, r_{i}}$ is the received signal-to-noise ratio (SNR) of the $m \rightarrow r_{i}$ link.

Let $R_{m, \Phi}^{B}$ be the achievable data rate at which $m$ can broadcast to all relays in $\Phi$. Successful decoding at all relays in $\Phi$ requires

$$
\begin{aligned}
R_{m, \Phi}^{B} & =\min _{\forall r_{i} \in \Phi} R_{m, r_{i}}=\log _{2}\left(1+\min _{\forall r_{i} \in \Phi} S N R_{m, r_{i}}\right) \\
& =\log _{2}\left(1+S N R_{m, \Phi}^{B}\right) .
\end{aligned}
$$

where $S N R_{m, \Phi}^{B} \triangleq \min _{\forall r_{i} \in \Phi} S N R_{m, r_{i}}$ represents the effective SNR of broadcast link $m \rightarrow \Phi$.

Maximal ratio combining (MRC) is used at the BS to combine the signals received from relays $r_{1} \sim r_{n}$ during the second half timeslot and the stored signal received from $m$ during the first half timeslot. Unlike distributed beamforming [15] where received amplitude is the sum of the signal amplitudes of all branches and thus perfect synchronization (i.e., frequency, phase, and time synchronizations) is needed, MRC does not require perfect synchronization and is typically seen in practical systems. With MRC, the combined SNR is the sum of the SNRs of all diversity branches. Consequently, successful decoding at the BS requires

$$
\begin{aligned}
R_{m, \Phi}^{C} & =\log _{2}\left(1+\frac{P_{m}\left|h_{m, B S}\right|^{2}+\sum_{\forall r_{i} \in \Phi} P_{r_{i}}\left|h_{r_{i}, B S}\right|^{2}}{N_{0} W}\right) \\
& =\log _{2}\left(1+S N R_{m, B S}+\sum_{\forall r_{i} \in \Phi} S N R_{r_{i}, B S}\right) \\
& =\log _{2}\left(1+S N R_{m, \Phi}^{C}\right) .
\end{aligned}
$$

where $S N R_{m, B S}$ and $S N R_{r_{i}, B S}$ are the received SNRs of the $m \rightarrow B S$ and $r_{i} \rightarrow B S$ links, and $S N R_{m, \Phi}^{C} \triangleq S N R_{m, B S}+$ $\sum_{\forall r_{i} \in \Phi} S N R_{r_{i}, B S}$ represents the effective SNR of cooperative link $(m, \Phi) \rightarrow B S$ after MRC.
Let $R_{m, B S}^{\Phi}$ be the achievable data rate from $m$ to the BS with the aid of relayset $\Phi$. We have

$$
R_{m, B S}^{\Phi}=\min \left(R_{m, \Phi}^{B}, R_{m, \Phi}^{C}\right) .
$$

\section{B. Problem Formulation}

The superior performance of PFS encourages the application of proportional fairness in relay-assisted cellular networks. Similar to the traditional PFS problem in a cellular network without relays, for a relay-assisted cellular network with the PF constraint, the objective is to maximize the overall logarithmic utility of all $(m, \Phi)$ pairs. This is formulated as follows

$$
\max \sum_{(m, \Phi) \in \Omega} \ln \left(\overline{\mu_{m, B S}^{\Phi}}\right) .
$$

s.t.,

$$
\begin{aligned}
I_{m, B S}^{\Phi}[t+1]=\left\{\begin{array}{ll}
1 & \text { if pair }(m, \Phi) \in \Omega \text { is scheduled } \\
0 & \text { else }
\end{array} .\right. \\
\mu_{m, B S}^{\Phi}[t+1]=\left(1-\frac{1}{k}\right) \mu_{m, B S}^{\Phi}[t] \\
+I_{m, B S}^{\Phi}[t+1] \times \frac{R_{m, B S}^{\Phi}[t+1]}{k} . \\
R_{m, B S}^{\Phi}[t+1]=\min \left(R_{m, \Phi}^{B}[t+1], R_{m, \Phi}^{C}[t+1]\right) .
\end{aligned}
$$

where $I_{m, B S}^{\Phi}[t+1]$ is the indicator function of the event that pair $(m, \Phi) \in \Omega$ is scheduled to transmit in slot $t+1$, $R_{m, B S}^{\Phi}[t]$ 's $k$-point moving average throughput (i.e., shortterm average throughput) up to time $t$ is denoted by $\mu_{m, B S}^{\Phi}[t]$, $\overline{\mu_{m, B S}^{\Phi}}=E\left[\mu_{m, B S}^{\Phi}\right]$ represents the long-term average throughput of pair $(m, \Phi)$

Equation (11) indicates that at each time slot, one node together with one relay set are selected for transmission at next time slot. The min operation in (13) accounts for the successful decoding required at both $\Phi$ and the BS.

\section{Proportionally Fair Cooperation (PFC)}

For the above optimization problem, we propose the following proportionally fair cooperation (PFC) algorithm.

As pointed out earlier, it has been proved that PFS maximizes the overall logarithmic utility of a cellular network by scheduling Node $j$ having the maximum PF metric $\frac{R_{j}[t+1]}{\mu_{j}[t]}$ [16]. Applying this to a relay-assisted cellular network, we have the solution to problem (10) by scheduling pair $(m, \Phi)$ having the maximum metric $\frac{R_{m, B S}^{\Phi}[t+1]}{\mu_{m, B S}^{\Phi}[t]}$. We call this a proportionally fair cooperation (PFC) algorithm, which is shown in Algorithm 1 in pseudo-code.

Algorithm 1 is similar to the traditional PFS except that it considers (node, relayset) pairs instead nodes. Specifically, lines $5-9$ are used to find one pair $\left(m^{*}, \Phi^{*}\right)$ with the maximum PF metric. Once $\left(m^{*}, \Phi^{*}\right)$ is found, the BS schedules Node $m^{*}$ and relayset $\Phi^{*}$ for two-phase transmission in next slot as illustrated in Fig. 1. Lines $11-14$ are then used to update the throughputs of all pairs using Equations (11) and (12). After that, the algorithm proceeds to next slot. 


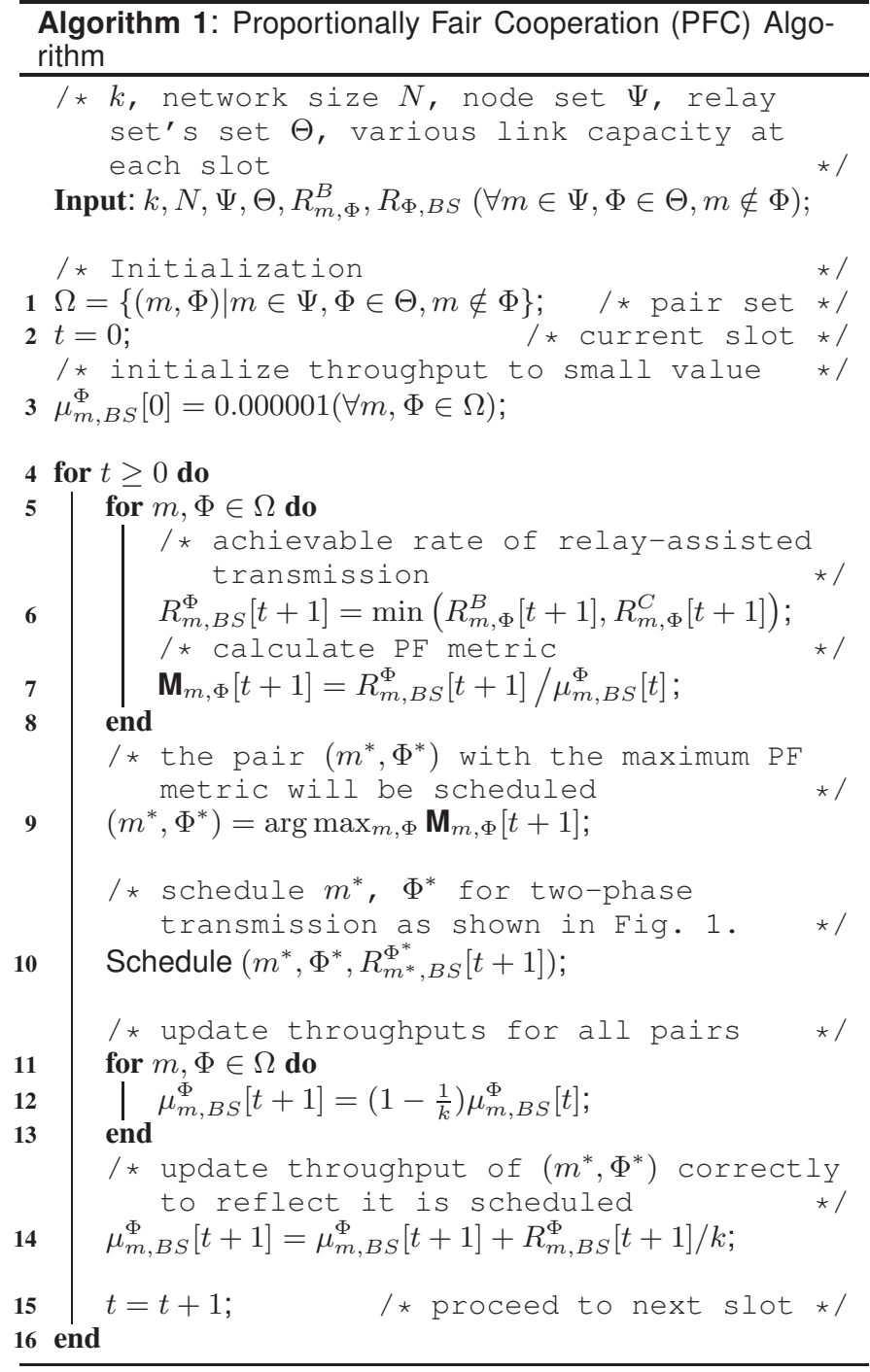

To implement PFC, the BS needs to know the channel state information (CSI) of both the $m \rightarrow B S$ and $m \rightarrow r_{i}$ links. Relay needs to know the CSI of the $m$ to relay link, which will be sent to the BS. We assume that the network operates in a slow fading scenario, so that channel estimation is possible and CSI is available at both the BS and relays.

The differences between PFC and PFS are: in an $N$-node cellular network, with PFS the BS will schedule $N$ nodes while with PFC the BS will schedule $N\left(\begin{array}{c}N-1 \\ n\end{array}\right)$ "nodes" (i.e., pairs); with PFS, the achievable rate of Node $m$ is estimated by the link quality of $m \rightarrow B S$, while with PFC, the achievable rate of the pair $(m, \Phi)$ should be estimated by the link quality of both $m \rightarrow \Phi$ and $(m, \Phi) \rightarrow B S$.

\section{Practical Consideration}

While PFC uses commercially available PFS algorithm and incorporates MRC to avoid the synchronization issue, we should point out that PFC may still suffer from the complexity issue as it has to enumerate $N\left(\begin{array}{c}N-1 \\ n\end{array}\right)(m, \Phi)$ pairs. When both $N$ and $n$ are large, PFC may not be feasible for practical implementation. In practice, we should limit $n$ (i.e., the size of relayset) to be 1 or 2 .
The complexity of PFC could be greatly reduced by decoupling the relayset selection from the scheduling algorithm. In other words, for each node $m$ in the network, one can assign a fixed relayset $\Phi_{m}$ to each node. Once the relaysets are assigned to all the nodes in the network, the PFS algorithm can be applied to obtain proportional fairness among these $\left(m, \Phi_{m}\right)$ pairs. The BS uses the same procedure as shown in Fig. 1 to MRC combine signals from both $m$ and $\Phi_{m}$ for successful reception. To differentiate from PFC, we call this decoupled method proportionally fair cooperation with fixed relays (PFC-FR). Obviously, PFC-FR only needs to schedule $N\left(m, \Phi_{m}\right)$ pairs. While complexity is remarkably reduced in PFC-FR, simulation (see Subsection IV-B) shows that, this decrease in complexity comes at a price of $11.4 \%$ decrease in throughput compared with PFC.

\section{ANALYSIS OF PFC OVER A RAYLEIGH FLAT FADING CHANNEL}

The proposed PFC algorithm presented in Subsection II-C is a solution for the optimization problem given by Equations (10)-(13). In this section, we analyze PFC over Rayleigh flat fading channels, and provide theoretical results. Specifically, we obtain a closed-form expression for the long-term throughput of each node for the PFC algorithm developed.

For the analysis, we use the following assumptions:

- Both direct and cooperative links are Rayleigh flat faded;

- The fading between any different links is mutually independent but not necessarily identical distributed;

- Channel fading keeps constant over each slot, and varies from slot to slot;

- Maximal ratio combining (MRC) is used at the BS to combine the signals from the source and relay nodes.

We considers pedestrian or fixed broadband access environments. According to [29], the channel can be considered as wide-sense stationary (WSS) as long as the mobile node moves within a range in the dimension of a few tens of the wavelength of the carrier signal, which corresponds to $\sim 10 \mathrm{~m}$ distance at $2.0 \mathrm{GHz}$ carrier frequency. At pedestrian speed, if each scheduling slot is $1 \mathrm{~ms}$, the channel can be considered as WSS in $\sim 10000$ slots. As such, the achievable rate $R$ of the channel is assumed WSS throughout the analysis.

To include the distance-dependent path loss, we use the model seen in [4], i.e.,, the instantaneous SNR is the multiplication of the average SNR (determined by path loss and shadowing ${ }^{1}$ ) with an randomly fading variable. This results in a single random variable that jointly models path loss and fading. Consequently, the instantaneous SNR of a Rayleigh fading link is modeled as an exponentially distributed random variable with a probability density function (pdf) given by

$$
\rho(x)=\frac{1}{\overline{S N R}} \times e^{-x / \overline{S N R}} .
$$

where $\overline{S N R}$ denotes the average SNR determined by the distance-dependent path loss.

We use $\overline{S N R_{m, r_{i}}}, \overline{S N R_{m, B S}}, \overline{S N R_{m, \Phi}^{B}}$, and $\overline{S N R_{m, \Phi}^{C}}$ to denote the average SNRs of the $m \rightarrow r_{i}, m \rightarrow B S, m \rightarrow \Phi$,

\footnotetext{
${ }^{1}$ Without loss of generality, shadowing is not considered here.
} 
and $(m, \Phi) \rightarrow B S$ links, respectively. As $S N R_{m, r_{i}}$ is an independent, exponentially distributed random variable, with $S N R_{m, \Phi}^{B} \triangleq \min _{\forall r_{i} \in \Phi} S N R_{m, r_{i}}$, the instantaneous SNR of broadcast link $m \rightarrow \Phi$ is also an exponentially distributed random variable, with a pdf given by

$$
\rho_{m, \Phi}^{B}(x)=\frac{1}{\overline{S N R_{m, \Phi}^{B}}} \times e^{-x / \overline{S N R_{m, \Phi}^{B}}} .
$$

where $\overline{S N R_{m, \Phi}^{B}}=1 / \sum_{i \in \Phi} \frac{1}{\overline{S N R_{m, r_{i}}}}$

With our setting, cooperative link is assumed Rayleigh faded. The pdf of $S N R_{m, \Phi}^{C}$, i.e., the SNR of the $(m, \Phi) \rightarrow B S$ link after MRC, is given by

$$
\rho_{m, \Phi}^{C}(x)=\frac{1}{\overline{S N R_{m, \Phi}^{C}}} \times e^{-x / \overline{S N R_{m, \Phi}^{C}}} .
$$

where $\overline{S N R_{m, \Phi}^{C}}=\overline{S N R_{m, B S}}+\sum_{\forall r_{i} \in \Phi} \overline{S N R_{r_{i}, B S}}$ is obtained as we are using MRC reception.

With (7) and (8), (9) can be re-written as

$$
\begin{aligned}
R_{m, B S}^{\Phi} & =\log _{2}\left(1+\min \left(S N R_{m, \Phi}^{B}, S N R_{m, \Phi}^{C}\right)\right) \\
& =\log _{2}\left(1+S N R_{m, B S}^{\Phi}\right) .
\end{aligned}
$$

where $S N R_{m, B S}^{\Phi} \triangleq \min \left(S N R_{m, \Phi}^{B}, S N R_{m, \Phi}^{C}\right)$ represents the effective SNR for the two-phase, relay-assisted transmission.

According to the above discussion, $S N R_{m, \Phi}^{B}$ and $S N R_{m, \Phi}^{C}$ are independent, exponentially distributed random variables. This means that $S N R_{m, B S}^{\Phi}$ is also an exponentially distributed random variable, with a pdf given by

$$
\begin{aligned}
& \rho_{m, B S}^{\Phi}(x)=\left(\frac{1}{\overline{S N R_{m, \Phi}^{B}}}+\frac{1}{\overline{S N R_{m, \Phi}^{C}}}\right) \times e^{\left(\frac{-x}{\overline{S N R_{m, \Phi}^{B}}}+\overline{\overline{S N R_{m, \Phi}^{C}}}\right)} \\
& =\left(\sum_{r_{i} \in \Phi} \frac{1}{\overline{S N R_{m, r_{i}}}}+\frac{1}{\overline{S N R_{m, B S}}+\sum_{r_{i} \in \Phi} \overline{S N R_{r_{i}, B S}}}\right)
\end{aligned}
$$

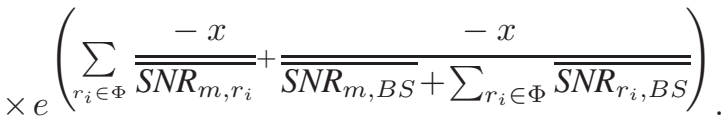

Obviously, the expectation of $S N R_{m, B S}^{\Phi}$ is given by

$$
\overline{S N R_{m, B S}^{\Phi}}=\frac{1}{\sum_{r_{i} \in \Phi} \frac{1}{\overline{S N R_{m, r_{i}}}}+\frac{1}{\overline{S N R_{m, B S}}+\sum_{r_{i} \in \Phi} \overline{S N R_{r_{i}, B S}}}} .
$$

We now use an interesting result on rate modeling for a Rayleigh fading channel. Smith and McKay etc. [30], [31] have shown that, in Rayleigh or Ricean fading networks, the achievable rate $R=\log _{2}[1+S N R]$ can be approximated modeled by a normally distributed random variable with extremely high accuracy. Specially, for a single-input-singleoutput (SISO) link over a Rayleigh flat fading channel, the Normal distribution is characterized by a mean and variance given as follows

$$
E[R]=\int_{0}^{\infty} e^{-x} \times \log _{2}(1+\overline{S N R} \times x) d x .
$$

$$
\sigma_{R}^{2}=\int_{0}^{\infty} e^{-x} \times\left(\log _{2}(1+\overline{S N R} \times x)\right)^{2} d x-(E[R])^{2} .
$$

Using this result, one can model $R_{m, B S}^{\Phi}$ as a normally distributed random variable, and we have the following theorem.

Theorem 1 In a Rayleigh flat fading cellular network with relay-assisted transmission, the achievable rate $R_{m, B S}^{\Phi}$ can be characterized by a normally distributed random variable.

Specifically, with (19), (20) and (21), we obtain the Normal distribution that characterizes $R_{m, B S}^{\Phi}$

$$
\begin{aligned}
& E\left[R_{m, B S}^{\Phi}\right]=\int_{0}^{\infty} e^{-x} d x \\
& \times \log _{2}\left(1+\frac{x}{\left.\sum_{r_{i} \in \Phi} \frac{1}{\overline{S N R_{m, r_{i}}}}+\frac{1}{\overline{S N R_{m, B S}}+\sum_{r_{i} \in \Phi} \overline{S N R_{r_{i}, B S}}}\right)}\right)
\end{aligned}
$$

$$
\begin{aligned}
& \sigma_{R_{m, B S}^{\Phi}}^{2}=-\left(E\left[R_{m, B S}^{\Phi}\right]\right)^{2}+\int_{0}^{\infty} e^{-x} d x \\
& \times\left(\log _{2}\left(1+\frac{x}{\left.\sum_{r_{i} \in \Phi} \frac{1}{\overline{S N R_{m, r_{i}}}}+\frac{1}{\overline{S N R_{m, B S}}+\sum_{r_{i} \in \Phi} \overline{S N R_{r_{i}, B S}}}\right)}\right)\right.
\end{aligned}
$$

With the above discussion for Rayleigh flat fading environments, the achievable rate of a node $m$ in a PFS cellular network and the capacity of a pair $(m, \Phi)$ in a PFC cellular network can both be characterized by Normal distribution.

In the following, the analysis starts with a PFS cellular network and then extends to a PFC cellular network using the above property for $R_{m, B S}^{\Phi}$. Note that some results on PFS can be found in our previous work [32]. The main drawback of the analysis in [32] is that we required (average throughput)/(average achievable rate) to be constant for all users to simply the analysis. This is in fact a unrealistic assumption. In this paper instead, we do not have such limitation and the analysis on PFS has been extended to study the proposed PFC algorithm. Interestingly, it is shown later that the analytical result on PFS is indeed a special case of that on PFC when there is no relay.

Before presenting our analytical results, we first provide some lemmas and corollaries.

\section{A. Lemmas and Corollaries}

Kelly [16] has provided the following formal definition of proportional fairness.

Definition 1 Let $s$ denote a user and $S$ the set of all users in the network. A vector of throughputs $x=\left(x_{s}, s \in S\right)$ is proportionally fair if it is feasible and if for any other feasible 
vector $x^{*}$, the aggregate of proportional changes is zero or negative:

$$
\sum_{s \in S}\left(x_{s}^{*}-x_{s}\right) / x_{s} \leq 0 .
$$

Regarding the achievable rate over a Rayleigh flat fading channel, we have the following two lemmas

Lemma 1 For a SISO or MIMO link over a Rayleigh flat fading channel, $\sigma_{R}$ w.r.t $E[R]$ is monotonically increasing, concave.

Proof: Please refer to Appendix A.

Lemma 2 In a PFS cellular network with Rayleigh flat fading, we have $\sigma_{R_{i}} / \sigma_{R_{j}} \leq E\left[\mu_{i}\right] / E\left[\mu_{j}\right] \leq E\left[R_{i}\right] / E\left[R_{j}\right]$, given $E\left[R_{j}\right] \leq E\left[R_{i}\right]$ for two users $i, j$.

Proof: Please refer to Appendix B.

Lemma 3 Let $Y_{k}(x)$ be a non-negative, monotonically increasing function of $x(k=1,2, \ldots, N)$, if it satisfies 1$)$ $x \geq 0$, and 2) $c_{i} / c_{j} \leq b_{i} / b_{j} \leq a_{i} / a_{j}\left(\forall a_{i} \geq a_{j}\right)$, with positive $a_{i}, a_{j}, b_{i}, b_{j}, c_{i}, c_{j}(\forall i, j=1,2, \ldots, N)$, we have

$$
\begin{gathered}
\prod_{\forall i \neq j, i=1}^{N} Y_{i}\left(\frac{b_{i}}{b_{j}} x\right) \leq \prod_{\forall i \neq j, a_{i} \geq a_{j}} Y_{i}\left(\frac{a_{i}}{a_{j}} x\right) \prod_{\forall i \neq j, a_{i}<a_{j}} Y_{i}\left(\frac{c_{i}}{c_{j}} x\right) . \\
\prod_{\forall i \neq j, i=1}^{N} Y_{i}\left(\frac{b_{i}}{b_{j}} x\right) \geq \prod_{\forall i \neq j, a_{i} \geq a_{j}} Y_{i}\left(\frac{c_{i}}{c_{j}} x\right) \prod_{\forall i \neq j, a_{i}<a_{j}} Y_{i}\left(\frac{a_{i}}{a_{j}} x\right) .
\end{gathered}
$$

Proof: Please refer to Appendix C.

We then have the following corollary,

Corollary 1 Let $Y_{k}(x)$ be a non-negative, monotonically increasing function of $x(k=1,2, \ldots, N)$, if it satisfies 1) $x \geq 0$, and 2) $c_{i} / c_{j} \leq b_{i} / b_{j} \leq a_{i} / a_{j}\left(\forall a_{i} \geq a_{j}\right)$, with positive $a_{i}, a_{j}, b_{i}, b_{j}, c_{i}, c_{j}(\forall i, j=1,2, \ldots, N)$, we have

$$
\begin{aligned}
\prod_{\forall i \neq j, i=1}^{N} Y_{i}\left(\frac{b_{i}}{b_{j}} x\right) \leq & \prod_{\forall i \neq j, a_{i}<a_{j}} Y_{i}\left(\frac{c_{i}}{c_{j}} x\right) \\
& \times \prod_{\forall i \neq j, a_{i} \geq a_{j}} Y_{i}\left(\frac{a_{i}}{a_{j}} x+a_{i}-\frac{c_{i}}{c_{j}} a_{j}\right) \\
\prod_{\forall i \neq j, i=1}^{N} Y_{i}\left(\frac{b_{i}}{b_{j}} x\right) \geq & \prod_{\forall i \neq j, a_{i} \geq a_{j}} Y_{i}\left(\frac{c_{i}}{c_{j}} x\right) \\
& \times \prod_{\forall i \neq j, a_{i}<a_{j}} Y_{i}\left(\frac{a_{i}}{a_{j}} x+a_{i}-\frac{c_{i}}{c_{j}} a_{j}\right)
\end{aligned}
$$

Proof: $c_{i} / c_{j} \leq a_{i} / a_{j}, \forall a_{i}>a_{j}, \Rightarrow \prod_{\forall i \neq j, a_{i} \geq a_{j}}^{N} Y_{i}\left(\frac{a_{i}}{a_{j}} x\right) \leq$ $\prod_{\forall i \neq j, a_{i} \geq a_{j}}^{N} Y_{i}\left(\frac{a_{i}}{a_{j}} x+a_{i}-\frac{c_{i}}{c_{j}} a_{j}\right)$, and $\prod_{\forall i \neq j, a_{i}<a_{j}}^{N} Y_{i}\left(\frac{a_{i}}{a_{j}} x\right) \geq$ $\prod_{\forall i \neq j, a_{i}<a_{j}}^{N} Y_{i}\left(\frac{a_{i}}{a_{j}} x+a_{i}-\frac{c_{i}}{c_{j}} a_{j}\right)$. Substituting into (24) and (25) finishes the proof.

\section{B. Theoretical Results for PFS and PFC}

In a cellular network with Rayleigh flat fading, since the achievable rate is modeled by a normally distributed random variable [30], [31], with Lemma 1, Lemma 2 and Corollary 1, we have the following theorem for PFS.

Theorem 2 (PFS Throughput) For an N-node PFS cellular network with Rayleigh flat fading, the long-term average throughput of Node $i$ is given by

$$
\begin{aligned}
E\left[\mu_{i}\right]= & \frac{E\left[R_{i}\right]}{N} \times\left(1-\left[\phi\left(-M_{i}\right)\right]^{N}\right) \\
& +\int_{-M_{i}}^{\infty} y \sigma_{i} \rho(y) \times[\phi(y)]^{N-1} d y .
\end{aligned}
$$

where $R_{i}$ and $\mu_{i}$ are the achievable rate and throughput of Node $i, E\left[R_{i}\right]$ and $\sigma_{i}$ denote the statistical average and standard deviation of $R_{i}, M_{i}=E\left[R_{i}\right] / \mu_{i}, \rho(\cdot)$ and $\phi(\cdot)$ are the $p d f$ and $c d f$ of zero mean, unit variance standard normal distribution.

Proof: Please refer to Appendix D.

We now have the important theoretical result for PFC.

Theorem 3 For an N-node PFC cellular network with Rayleigh flat fading, the long-term average throughput of Node $m$ is given by

$$
\begin{aligned}
& E\left[\mu_{m}\right]=\sum_{\Phi \in \Theta, m \notin \Phi} E\left[\mu_{m, B S}^{\Phi}\right] \\
& =\sum_{\Phi \in \Theta, m \notin \Phi}\left(\begin{array}{c}
\frac{E\left[R_{m, B S}^{\Phi}\right]}{N\left(\begin{array}{c}
N-1 \\
n
\end{array}\right)} \times\left(1-\left[\phi\left(-M_{m, \Phi}\right)\right]^{N(N-1)}\right. \\
+\int_{-M_{m, \Phi}}^{\infty} y \sigma_{R_{m, B S}^{\Phi}}^{\Phi} \rho(y) \times[\phi(y)]^{N\left(\begin{array}{c}
N-1 \\
n
\end{array}\right)-1} d y
\end{array}\right) .
\end{aligned}
$$

where $E\left[R_{m, B S}^{\Phi}\right]$ and $\sigma_{R_{m, B S}^{\Phi}}$ denote the statistical average and standard deviation of $R_{m, B S}^{\Phi}, \quad M_{m, \Phi}=$ $E\left[R_{m, B S}^{\Phi}\right] / \sigma_{R_{m, B S}^{\Phi}}$.

Proof: For the given cellular network, there are $N\left(\begin{array}{c}N-1 \\ n\end{array}\right)$ possible pairs. In the beginning of this section, we have proved that in a Rayleigh fading cellular network with relay-assisted transmission, the achievable rate $R_{m, B S}^{\Phi}$ of pair $(m, \Phi)$ can be modeled as a normally distributed random variable (Theorem 1). With Theorem 2, we have

$$
\begin{aligned}
E\left[\mu_{m, B S}^{\Phi}\right] & =\frac{E\left[R_{m, B S}^{\Phi}\right]}{N\left(\begin{array}{c}
N-1 \\
n
\end{array}\right)} \times\left(1-\left[\phi\left(-M_{m, \Phi}\right)\right]^{N\left(\begin{array}{c}
N-1 \\
n
\end{array}\right)}\right) \\
& +\int_{-M_{m, \Phi}}^{\infty} y \sigma_{R_{m, B S}^{\Phi}} \rho(y) \times[\phi(y)]^{N\left(\begin{array}{c}
N-1 \\
n
\end{array}\right)-1} d y .
\end{aligned}
$$

where $E\left[R_{m, B S}^{\Phi}\right]$ and $\sigma_{R_{m, B S}^{\Phi}}$ are given by (22) and (23).

Since $E\left[\mu_{m}\right]=\sum_{\Phi \in \Theta, m \notin \Phi} E\left[\mu_{m, B S}^{\Phi}\right]$, Theorem 3 follows immediately.

One can justify that (27) reduces to (26) if no relay is used (i.e., $\Phi$ has $n=0$ relay). In such case, PFC is indeed PFS.

With Theorems 2 and 3, we have the following corollary. 
Corollary 2 For Node $m$ in a Rayleigh fading cellular network, the throughput gain of PFC over PFS is

$$
G_{m}=\frac{\sum_{\Phi \in \Theta, m \notin \Phi}\left(\begin{array}{l}
\frac{E\left[R_{m, B S}^{\Phi}\right]}{N\left(\begin{array}{c}
N-1 \\
n
\end{array}\right)} \times\left(1-\left[\phi\left(-M_{m, \Phi}\right)\right]^{N\left(\begin{array}{c}
N-1 \\
n
\end{array}\right)}\right. \\
+\int_{-M_{m, \Phi}}^{\infty} y \sigma_{R_{m, B S}^{\Phi}}^{\Phi} \rho(y) \times[\phi(y)]^{N\left(\begin{array}{c}
N-1 \\
n
\end{array}\right)-1} d y
\end{array}\right)}{\left(\begin{array}{l}
\frac{E\left[R_{m}\right]}{N} \times\left(1-\left[\phi\left(-M_{m}\right)\right]^{N}\right) \\
+\int_{-M_{m}}^{\infty} y \sigma_{m} \rho(y) \times[\phi(y)]^{N-1} d y
\end{array}\right)}
$$

Remark 1 Numerical calculations show that $\left(\phi\left(-M_{m}\right)\right)^{N} \ll 1, \quad\left(\phi\left(-M_{m, \Phi}\right)\right)^{N(N-1)} \ll 1$, $\int_{-M_{m}}^{\infty} y \rho(y)[\phi(y)]^{N-1} d y \quad \approx \quad \int_{-\infty}^{\infty} y \rho(y)[\phi(y)]^{N-1} d y$, $\int_{-M_{m, \Phi}}^{\infty} y \rho(y)[\phi(y)]^{N\left(\begin{array}{c}N-1 \\ n\end{array}\right)-1} d y \approx \int_{-\infty}^{\infty} y \rho(y)[\phi(y)]^{N\left(\begin{array}{c}N-1 \\ n\end{array}\right)-1} d y$, so Theorem 2, Theorem 3 and Corollary 2 reduce to the following forms,

$$
\begin{gathered}
E\left[\mu_{i}\right]=\frac{E\left[R_{i}\right]}{N}+\sigma_{i} \int_{-\infty}^{\infty} y \rho(y)[\phi(y)]^{N-1} d y . \\
E\left[\mu_{m}\right]=\frac{E^{C}\left[R_{m}\right]}{N}+\left(\begin{array}{c}
N-1 \\
n
\end{array}\right) \sigma_{R_{m}}^{C} \int_{-\infty}^{\infty} y \rho(y)[\phi(y)]^{N\left(\begin{array}{c}
N-1 \\
n
\end{array}\right)} d y . \\
G_{m}=\frac{\frac{E^{C}\left[R_{m}\right]}{N}+\left(\begin{array}{c}
N-1 \\
n
\end{array}\right) \sigma_{R_{m}}^{C} \int_{-\infty}^{\infty} y \rho(y)[\phi(y)]^{N\left(\begin{array}{c}
N-1 \\
n
\end{array}\right)} d y}{\frac{E\left[R_{m}\right]}{N}+\sigma_{m} \int_{-\infty}^{\infty} y \rho(y)[\phi(y)]^{N-1} d y} .
\end{gathered}
$$

where $E^{C}\left[R_{m}\right] \triangleq \sum_{\Phi \in \Theta, m \notin \Phi} E\left[R_{m, B S}^{\Phi}\right] /\left(\begin{array}{c}N-1 \\ n\end{array}\right), \sigma_{R_{m}}^{C} \triangleq$ $\sum_{\Phi \in \Theta, m \notin \Phi} \sigma_{R_{m, B S}^{\Phi}} /\left(\begin{array}{c}N-1 \\ n\end{array}\right)$.

In PFC, since there are $\left(\begin{array}{c}N-1 \\ n\end{array}\right)$ possible relaysets for each node $m, E^{C}\left[R_{m}\right]$ and $\sigma_{R_{m}}^{C}$ indeed represent the average and standard deviation of the achievable rate of Node $m$ with the help of relaying. According to (20) and (21), $E[R]$ and $\sigma_{R}$ are both increasing functions of SNR. With MRC reception, the received SNR is increased. Hence for any relayset $\Phi$, we have $E\left[R_{m, B S}^{\Phi}\right]>E\left[R_{m}\right]$ and $\sigma_{R_{m, B S}^{\Phi}}>\sigma_{R_{m}}$, where $E\left[R_{m}\right]$, $\sigma_{R_{m}}$ represent values without relaying. Obviously $E^{C}\left[R_{m}\right]>$ $E\left[R_{m}\right], \sigma_{R_{m}}^{C}>\sigma_{R_{m}}$.

Define $A(K) \triangleq K \int_{-\infty}^{\infty} y \rho(y)[\phi(y)]^{K-1} d y(K=1,2, \ldots)$, (30)-(32) are re-written as

$$
\begin{gathered}
E\left[\mu_{i}\right]=\frac{E\left[R_{i}\right]+A(N) \sigma_{R_{i}}}{N} . \\
E\left[\mu_{m}\right]=\frac{E^{C}\left[R_{m}\right]+A\left(N\left(\begin{array}{c}
N-1 \\
n
\end{array}\right)\right) \sigma_{R_{m}}^{C}}{N} . \\
G_{m}=\frac{E^{C}\left[R_{m}\right]+A\left(N\left(\begin{array}{c}
N-1 \\
n
\end{array}\right)\right) \sigma_{R_{m}}^{C}}{E\left[R_{i}\right]+A(N) \sigma_{R_{i}}} .
\end{gathered}
$$

For reference, Fig. 2 plots $A(K)$ for various $K$. In the case when relaying helps little, we have $E^{C}\left[R_{m}\right] \approx E\left[R_{m}\right]$, $\sigma_{R_{m}}^{C} \approx \sigma_{R_{m}}$. Even for such case, we still have $G_{m}>1$ since $A(K)$ is increasing function of $K$. In this case, PFC mainly benefits from multi-user diversity. In the case when relaying does help, we will have larger $G_{m}$, which comes from both

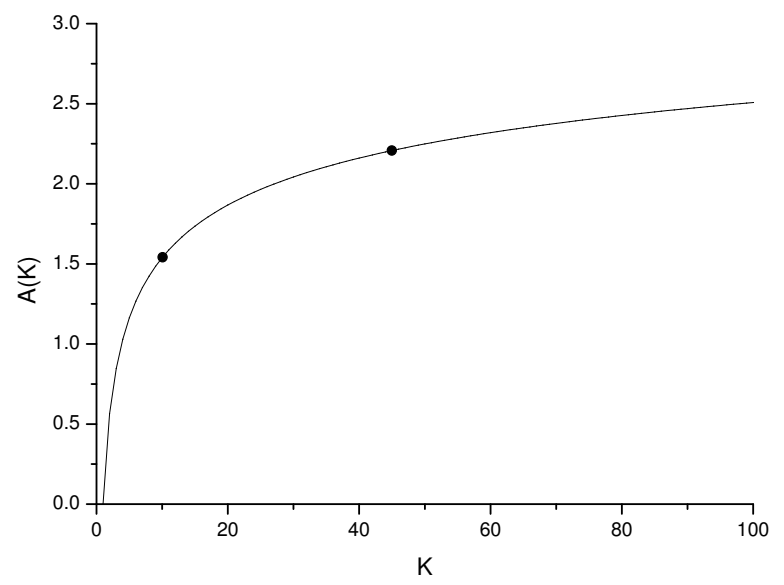

Fig. 2. $\mathrm{A}(\mathrm{K})$ w.r.t $\mathrm{K}$

larger $A(K)$ and larger $E^{C}\left[R_{m}\right], \sigma_{R_{m}}^{C}$. In other words, PFC benefits from both cooperative diversity (larger average and standard deviation of achievable rate due to relaying) and multi-user diversity (larger $A(K)$ due to larger $K)^{2}$.

Corollary 2 provides a closed-form expression for quantifying the throughput gain with relay-assisted transmission for cellular networks under the PF constraint.

\section{Numerical and Simulation Results}

Numerical experiment and simulation are conducted to evaluate the PFC algorithm.

As mentioned in Section III, we use the method in [4] to model the instantaneous SNR, i.e., the instantaneous SNR is modeled as an exponentially distributed random variable with the mean SNR determined by the distance-dependent path loss. The path loss (in $\mathrm{dB}$ ) at distance $d$ is modeled by

$$
P L_{d}=P L_{d_{0}}+\alpha \times 10 \log _{10}\left(d / d_{0}\right) .
$$

where $\alpha$ is path loss exponent, and $P L_{d_{0}}$ is the path loss at reference distance $d_{0}$.

The average SNR (in $\mathrm{dB}$ ) at $d$ is then modeled as

$$
\overline{S N R_{d}}=\overline{S N R_{d_{0}}}-\alpha \times 10 \log _{10}\left(d / d_{0}\right) .
$$

where $\overline{S N R_{d_{0}}}$ is the average $S N R$ at reference distance $d_{0}$.

We first performed numerical experiment to compare the throughput performance of PFC and PFS over a Rayleigh flat fading channel.

\section{A. Numerical Experiment}

In the numerical experiment, we use the following settings: 10 nodes $n_{1} \sim n_{10}$ are placed in an area of $1.2 \mathrm{~km} \times 1.6 \mathrm{~km}$ as shown in Fig. 3. Path loss exponent $\alpha=2.5 \sim 4.5$ for various environments, reference distance $d_{0}=100 \mathrm{~m}$, average SNR at $d_{0}$ is $\overline{S N R_{d_{0}}}=30 \mathrm{~dB}$.

Equations (20) and (21) are used to calculate the mean and standard deviation of the achievable rate in direct transmission; (22) and (23) are used to calculate the mean and standard

\footnotetext{
${ }^{2}$ Though some researchers may view cooperative diversity as another form of multi-user diversity, here we differentiate it from multi-user diversity for ease of exposition.
} 


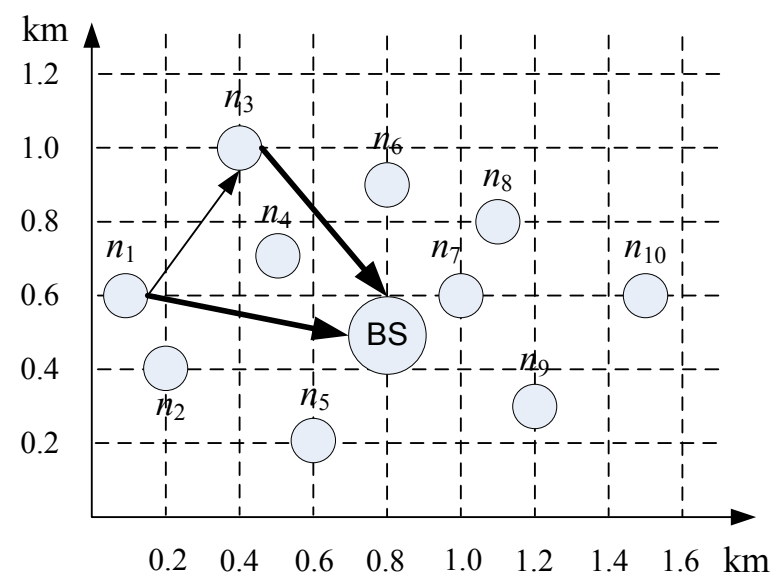

Fig. 3. Network Topology: a 10-node cellular network

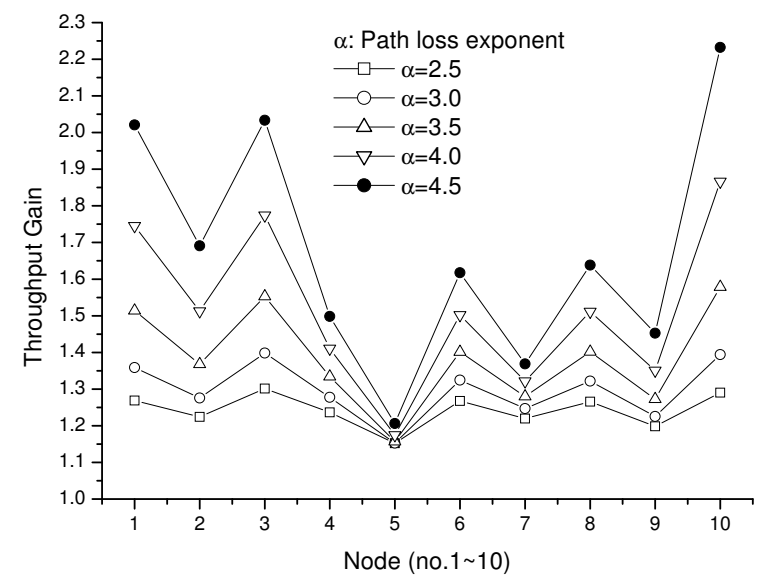

Fig. 4. PFC vs. PFS: theoretical result - a throughput comparison

deviation of the achievable rate in relay-assisted transmission. Finally, we use (33), (34) and (35) to evaluate the performance of PFS and PFC.

We use a configuration of $n=1$ (i.e., a relay set $\Phi$ contains only one relay). We plot in Fig. 4 the relaying gain $G_{m}$ for each node under various path loss scenarios. We can see that with relay-assisted transmission, PFC significantly improves performance. Fig. 4 also shows that the PFC performance increases with path loss exponent and bad-channel nodes $n_{1}, n_{2}, n_{3}, n_{10}$ benefit more from PFC than good-channel nodes $n_{4} \sim n_{9}$. These indicate that PFC will perform better in relatively bad communication environments.

In Fig. 4, we notice Node $n_{5}$ has relatively small gain. This is due to two reasons: 1 ). Node $n_{5}$ locates near to the BS and already has relatively good channel condition. This makes relaying less efficient; 2). All possible DF relays are far from Node $n_{5}$. This means that the received SNRs may not be high enough to allow successful decoding at the DF relays, which in turn reduces the relaying gain.

\section{B. Simulation}

Using the configuration given in Subsection IV-A, we evaluate the accuracy of our theoretical models by simulation. The PFC algorithm presented in Subsection II-C is used in

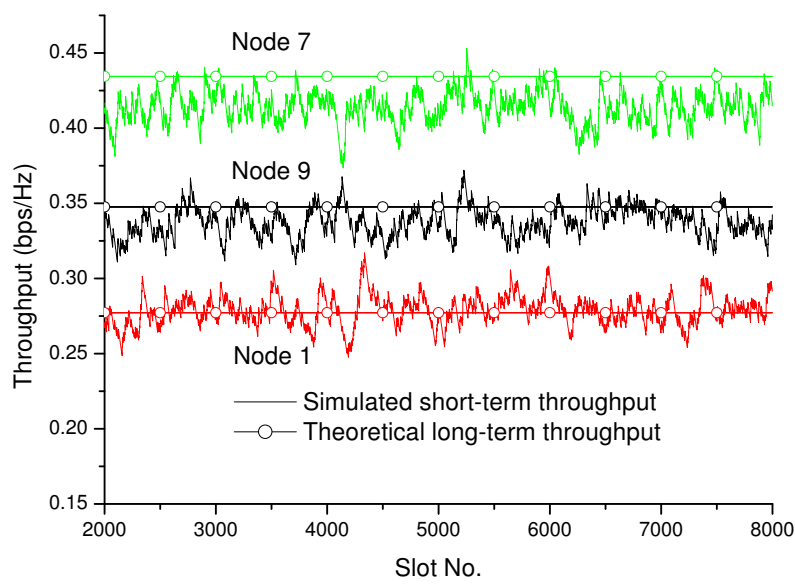

Fig. 5. PFC for the worst/medium/best node: Simulation vs. Analysis

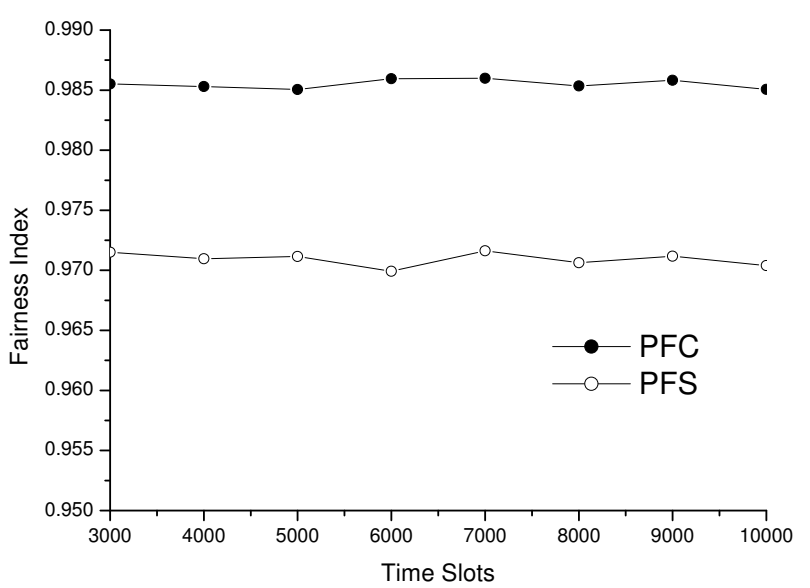

Fig. 6. Fairness index: PFC vs. PFS

simulations. Path loss exponent is 3.0. Moving average factor $k=500$. The simulation runs for 8000 slots. Fig. 5 depicts the throughput curves from simulation and theoretical analysis for $n_{1}, n_{7}$, and $n_{9}$, which represent the worst-, the medium-, and the best-channel nodes in the network.

The validity of our theoretical results is illustrated in Fig. 5 where the solid lines are the short-term throughput from simulation and the circle ones are the long-term throughput from analysis. We can see that the analytic results match well with the simulation ones.

We also evaluate the fairness of PFC. As a metric of fairness measurement, we use Jain's fairness index [33], which is defined as $f\left(x_{1}, x_{2}, \ldots, x_{N}\right)=\left(\sum_{i=1}^{N} x_{i}\right)^{2} /\left(N \sum_{i=1}^{N} x_{i}^{2}\right)$ where $x_{i}$ represents the long-term average throughput of User $i$. In particular, $f=1$ corresponds to optimal fairness, and that the larger is $f$, the more fair are the algorithm in allocating throughput to the users.

In our simulations, PFC exhibits very nice fairness, with a fairness index $f \approx 0.985$, as shown in Fig. 6 . We notice that PFC has a higher fairness performance than PFS. This can be interpreted as "correlation increases fairness" or "correlation reduces throughput". In simulations for PFS, user channels are independent and hence uncorrelated. If no correlation exists between relay-assisted user channels, PFC will have the same 
fairness as PFS since they are using the same PF metric. However, as different nodes could be served by the same relay, user channels are indeed correlated in PFC, resulting in degraded throughput performance (compared with that of PFC when no correlation is assumed $)^{3}$, thus increased fairness due to throughput-fairness tradeoff.

As mentioned earlier in Subsection II-D, the complexity of PFC can be considerably reduced by using the decoupling PFC-FR method. We are interested in the complexity and performance trade-off. In the simulation for PFC-FR, for each node $m$, an optimum relayset $\Phi_{m}$ is selected to be the one that maximizes the average effective SNR of Node $m$, i.e., $\Phi_{m}=\arg _{\Phi} \max \overline{S N R_{m, B S}^{\Phi}}$, where $\overline{S N R_{m, B S}^{\Phi}}$ is determined by (19). We use the same configuration as before. For the network shown in Fig. 3, simulations reveal that the overall throughputs of PFC, PFC-FR, and PFS are $3.5 \mathrm{bps} / \mathrm{Hz}, 3.1 \mathrm{bps} / \mathrm{Hz}$, and $2.7 \mathrm{bps} / \mathrm{Hz}$, respectively. Not surprisingly, both PFC and PFCFR have higher throughput than PFS because of cooperative diversity. The result also indicates that, compared with PFC, the reduced complexity in PFC-FR comes at a price of about $11.4 \%$ decrease in throughput, which comes from reduced multi-user diversity.

\section{CONCLUSiON}

Relay-assisted transmission in cellular networks is a practical cooperative method that does not require complex code design to benefit from the spatial diversity. Adding proportional fairness into cooperation, we proposed the proportionally fair cooperation (PFC) algorithm for cellular networks. This technique uses traditional MRC reception and thus does not have synchronization issue typically seen in network MIMO or distributed beamforming systems.

To facilitate research, we further analyzed PFC and presented a framework to quantify its performance. The ODE analysis, typically used to study PFS in cellular networks or CPF in multi-base-station systems, is not feasible for PFC. Using results on rate modeling for Rayleigh fading channels, we provided closed-form expressions to evaluate the performance of PFC, without turning to the time-consuming ODE analysis. Though this paper mainly talks about PFC, the theoretical results presented for the PFC algorithm can also be used to evaluate PFS where there is no relay.

Both simulation and numerical results have validated that PFC significantly improves the throughput performance by utilizing both cooperative diversity and multi-user diversity, while at the same time exhibit very good fairness among users by adding correlation to user channels. The increased performance of PFC over PFS comes with additional overhead: To implement PFC, the BS needs to collect the CSI of both $m$ to the BS and $m$ to relay links. Relay needs to know the CSI of the $m$ to relay link, which is sent to the BS via a control/feedback channel (or in piggy-back manner). In PFS, the BS only needs to know the CSI of $N$ links, while the BS in PFC needs to know the CSI of $N+N \times \frac{N-1}{2}=N \times \frac{N+1}{2}$

\footnotetext{
${ }^{3}$ For the simplest case that channels are fully correlated, one can easily apply the ODE analysis to show that both PFC and PFS reduce to roundrobin (RR) scheduling, which does not have the multi-user diversity gain.
}

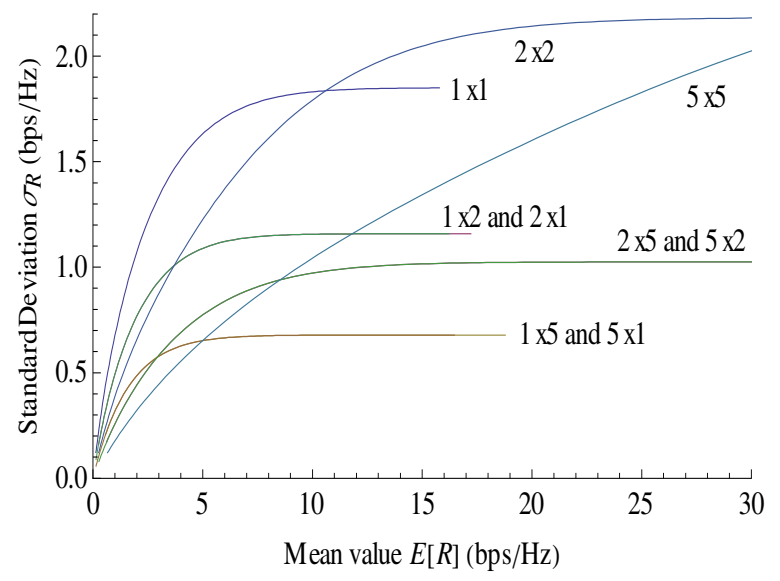

Fig. 7. $\sigma_{R}$ vs. $E[R]$ : Rayleigh flat fading SISO/MIMO channel

links; the CSI of $N \times \frac{N-1}{2} m$ to relay links should be sent to the BS. Considering the complexity, we should limit the size of relayset to be 1 or 2 for PFC. Alternatively, one can use the decoupling PFC-FR method to reduce implementation complexity, at a price of about $11.4 \%$ decrease in throughput.

\section{ACKNOWLEDGEMENT}

This work was supported, in part, by Johns Hopkins University, Applied Physics Laboratory's internal research and development funds.

\section{APPENDIX A}

PROOF OF LEMMA 1

Equations (20) and (21) provide the mean and variance of $R$ for a Rayleigh fading SISO channel, respectively. For the $t \times r$ MIMO communication between two peers where $t$ and $r$ are the number of transmit and receive antenna, we have [30], [34]

$$
\begin{aligned}
E[R]= & \int_{0}^{\infty} \omega(\lambda) \sum_{k=0}^{m-1} \frac{k ! \lambda^{n-m} e^{-\lambda}}{(k+n-m) !}\left[L_{k}^{n-m}(\lambda)\right]^{2} d \lambda . \\
\sigma_{R}^{2}= & \int_{0}^{\infty} \omega^{2}(\lambda) \sum_{k=0}^{m-1} \frac{k ! \lambda^{n-m} e^{-\lambda}}{(k+n-m) !}\left[L_{k}^{n-m}(\lambda)\right]^{2} d \lambda \\
& -\sum_{i=0}^{m-1} \sum_{j=0}^{m-1}\left[\frac{i ! j !}{(i+n-m) !(j+n-m) !}\right] \\
& \times\left(\int_{0}^{\infty} \lambda^{n-m} e^{-\lambda} L_{i}^{n-m}(\lambda) L_{j}^{n-m}(\lambda) \omega(\lambda)\right)^{2} .
\end{aligned}
$$

where $\omega(\lambda)=\log _{2}(1+\overline{S N R} \cdot \lambda / t), m=\min (t, r), n=\max (t, r)$, $L_{k}^{n-m}(\cdot)$ is generalized Laguerre polynomials of order $k$.

With (20), (21), (38) and (39), one can prove $\frac{d \sigma_{R}}{d E[R]}>0$ and $\frac{1}{d E[R]}\left(\frac{\sigma_{R}}{d E[R]}\right)<0$ for both SISO and MIMO cases, i.e., $\sigma_{R}$ w.r.t $E[R]$ is monotonically increasing, concave. For reference, we plot in Fig. 7 the curves $\sigma_{R}$ vs. $E[R]$ to show the concavity for both SISO and MIMO cases. 


\section{APPENDIX B}

PROOF OF LEMMA 2

In wireless networks, both channel fluctuation (i.e., $\sigma_{R}$ ) and average channel quality (i.e., $E[R]$ ) contribute to average throughput. Without lost of generality, a very small increase in average throughput can be written as $\Delta E\left[\mu^{*}\right]=f^{*} \cdot E[R]$. $\Delta \overline{S N R}+g^{*} \cdot \sigma_{R} \cdot \Delta \overline{S N R}$ where $f^{*}>0, g^{*}>0$ represent the weights of $E[R]$ and $\sigma_{R}$, respectively.

In an $N$-user cellular network, let say users $i, j$ are provided average throughputs of $E\left[\mu_{i}\right]$ and $E\left[\mu_{j}\right]$ when PFS is used. We assume $E\left[\mu_{i}\right] \geq E\left[\mu_{j}\right]$.

If a scheduling algorithm other than PFS is able to increase $E\left[\mu_{i}\right]$ to $E\left[\mu_{i}\right]+\Delta E\left[\mu_{i}^{*}\right]$ and decrease $E\left[\mu_{j}\right]$ to $E\left[\mu_{j}\right]-\Delta E\left[\mu_{j}^{*}\right]$, while keeping other users' average throughputs unchanged, according to the definition of proportional fairness (Definition 1), we have

$$
\frac{\Delta E\left[\mu_{j}^{*}\right]}{E\left[\mu_{j}\right]} \geq \frac{\Delta E\left[\mu_{i}^{*}\right]}{E\left[\mu_{i}\right]}
$$

Lemma 1 shows that $\frac{\sigma_{R_{i}}}{E\left[R_{i}\right]} \leq \frac{\sigma_{R_{j}}}{E\left[R_{j}\right]}$. Since $\frac{\Delta E\left[\mu_{i}^{*}\right]}{\Delta E\left[\mu_{j}^{*}\right]}=$ $\frac{f^{*} \cdot E\left[R_{i}\right]+g^{*} \cdot \sigma_{R_{i}}}{f^{*} \cdot E\left[R_{j}\right]+g^{*} \cdot \sigma_{R_{j}}}$, we have

$$
\frac{\Delta E\left[\mu_{i}^{*}\right]}{\Delta E\left[\mu_{j}^{*}\right]} \geq \frac{\sigma_{R_{i}}}{\sigma_{R_{j}}}
$$

Combining (40) and (41), we obtain

$$
\frac{E\left[\mu_{i}\right]}{E\left[\mu_{j}\right]} \geq \frac{\sigma_{R_{i}}}{\sigma_{R_{j}}}
$$

Similarly, if a scheduling algorithm is able to decrease $E\left[\mu_{i}\right]$ to $E\left[\mu_{i}\right]-\Delta E\left[\mu_{i}^{*}\right]$ and increase $E\left[\mu_{j}\right]$ to $E\left[\mu_{j}\right]+\Delta E\left[\mu_{j}^{*}\right]$, while keeping other users' average throughputs unchanged, with Definition 1 we can prove

$$
\frac{E\left[\mu_{i}\right]}{E\left[\mu_{j}\right]} \leq \frac{E\left[R_{i}\right]}{E\left[R_{j}\right]}
$$

Putting together (42) and (43) completes the proof.

\section{APPENDIX C}

PROOF OF LEMMA 3

Let $B=\prod_{\forall i \neq j, i=1}^{N} Y_{i}\left(b_{i} x / b_{j}\right)$. Since $c_{i} / c_{j} \leq b_{i} / b_{j} \leq$ $a_{i} / a_{j}\left(\forall a_{i}>a_{j}\right)$, for non-negative, monotonically increasing $Y_{k}(\cdot)(\forall k=1,2, \ldots, N)$, we have

$$
\begin{aligned}
B^{2} \leq & \prod_{\forall i \neq j, a_{i} \geq a_{j}} Y_{i}\left(\frac{a_{i}}{a_{j}} x\right)_{\forall i \neq j, a_{i}<a_{j}} \prod_{i}\left(\frac{b_{i}}{b_{j}} x\right) \\
\times & \prod_{\forall i \neq j, a_{i} \geq a_{j}} Y_{i}\left(\frac{b_{i}}{b_{j}} x\right)_{\forall i \neq j, a_{i}<a_{j}} \prod_{i}\left(\frac{c_{i}}{c_{j}} x\right) \\
= & B \times \prod_{\forall i \neq j, a_{i} \geq a_{j}} Y_{i}\left(\frac{a_{i}}{a_{j}} x\right)_{\forall i \neq j, a_{i}<a_{j}} \prod_{i} Y_{i}\left(\frac{c_{i}}{c_{j}} x\right) \\
B^{2} & \prod_{\forall i \neq j, a_{i} \geq a_{j}} Y_{i}\left(\frac{c_{i}}{c_{j}} x\right)_{\forall i \neq j, a_{i}<a_{j}} \prod_{i}\left(\frac{b_{i}}{b_{j}} x\right) \\
\times & \prod_{\forall i \neq j, a_{i} \geq a_{j}} Y_{i}\left(\frac{b_{i}}{b_{j}} x\right)_{\forall i \neq j, a_{i}<a_{j}} \prod_{i}\left(\frac{a_{i}}{a_{j}} x\right) \\
= & B \times \prod_{\forall i \neq j, a_{i} \geq a_{j}} Y_{i}\left(\frac{c_{i}}{c_{j}} x\right)_{\forall i \neq j, a_{i}<a_{j}} \prod_{i} Y_{i}\left(\frac{a_{i}}{a_{j}} x\right)
\end{aligned}
$$

This concludes the proof.

\section{APPENDIX D \\ PROOF OF THEOREM 2}

Applying Bayes's theorem, we write (1) as

$$
\begin{aligned}
& E\left[\mu_{j}[t]\right]=E\left[R_{j}[t+1] \mid I_{j}[t+1]=1\right] \operatorname{Pr}\left(I_{j}[t+1]=1\right) \\
& =\operatorname{Pr}\left(I_{j}[t+1]=1\right) \int_{0}^{\infty} x f_{R_{j}}\left(x \mid I_{j}[t+1]=1\right) d x \\
& =\int_{0}^{\infty} x f_{R_{j}}(x) \operatorname{Pr}\left(I_{j}[t+1]=1 \mid R_{j}[t+1]=x\right) d x
\end{aligned}
$$

where $\operatorname{Pr}\left(I_{j}[t+1]=1\right)$ is the average probability that $j$ will be scheduled in slot $t+1, \operatorname{Pr}\left(I_{j}[t+1]=1 \mid R_{j}[t+1]=x\right)$ is the conditional probability. Kushner [17] has proved that $\mu_{j}[t]$ converges to $E\left[\mu_{j}\right]$. So we have

$$
\begin{aligned}
\operatorname{Pr}\left(I_{j}[t+1]=\right. & \left.1 \mid R_{j}[t+1]=x\right) \\
& =\operatorname{Pr}\left(\forall i \neq j, \frac{R_{i}[t+1]}{\mu_{i}}<\frac{x}{\mu_{j}}\right) \\
& =\operatorname{Pr}\left(\forall i \neq j, \frac{R_{i}[t+1]}{E\left[\mu_{i}\right]}<\frac{x}{E\left[\mu_{j}\right]}\right) .
\end{aligned}
$$

Assuming WSS $R$, we rewrite (44) as

$E\left[\mu_{j}\right]=\int_{0}^{\infty} x f_{R_{j}}(x) \prod_{\forall i \neq j, i=1}^{N} F_{R_{i}}\left(E\left[\mu_{i}\right] \times x / E\left[\mu_{j}\right]\right) d x$

Lemma 1, Lemma 2, Corollary 1 together with (46) yield

$$
\begin{aligned}
E\left[\mu_{j}\right] \leq & \sigma_{R_{j}} \int_{-M_{j}}^{\infty}\left(y \sigma_{R_{j}}+E\left[R_{j}\right]\right) f_{R_{j}}\left(y \sigma_{R_{j}}+E\left[R_{j}\right]\right) \\
& \times \prod_{\forall i \neq j, E\left[R_{i}\right] \geq E\left[R_{j}\right]}^{N} F_{R_{i}}\left(y \frac{E\left[R_{i}\right]}{E\left[R_{j}\right]} \sigma_{R_{j}}+E\left[R_{i}\right]\right) \\
& \times \prod_{\forall i \neq j, E\left[R_{i}\right]<E\left[R_{j}\right]}^{N} F_{R_{i}}\left(y \sigma_{R_{i}}+\frac{\sigma_{R_{i}}}{\sigma_{R_{j}}} E\left[R_{j}\right]\right) d y .(47)
\end{aligned}
$$

$$
\begin{aligned}
E\left[\mu_{j}\right] \geq \sigma_{R_{j}} \int_{-M_{j}}^{\infty}\left(y \sigma_{R_{j}}+E\left[R_{j}\right]\right) f_{R_{j}}\left(y \sigma_{R_{j}}+E\left[R_{j}\right]\right) \\
\times \prod_{\forall i \neq j, E\left[R_{i}\right]<E\left[R_{j}\right]}^{N} F_{R_{i}}\left(y \frac{E\left[R_{i}\right]}{E\left[R_{j}\right]} \sigma_{R_{j}}+E\left[R_{i}\right]\right) \\
\times \prod_{\forall i \neq j, E\left[R_{i}\right] \geq E\left[R_{j}\right]}^{N} F_{R_{i}}\left(y \sigma_{R_{i}}+\frac{\sigma_{R_{i}}}{\sigma_{R_{j}}} E\left[R_{j}\right]\right) d y .(48)
\end{aligned}
$$

One can prove that the following expression lies between the upper and lower bounds given in (47) and (48),

$$
\begin{array}{r}
\sigma_{R_{j}} \int_{-M_{j}}^{\infty}\left(y \sigma_{R_{j}}+E\left[R_{j}\right]\right) f_{R_{j}}\left(y \sigma_{R_{j}}+E\left[R_{j}\right]\right) \\
\times \prod_{\forall i \neq j, i=1}^{N} F_{R_{i}}\left(y \sigma_{R_{i}}+E\left[R_{i}\right]\right) d y .
\end{array}
$$

We then use (49) to estimate $E\left[\mu_{j}\right]$. For normally distributed $R_{i}, f_{R_{i}}(x)=\rho\left(\left(x-E\left[R_{i}\right]\right) / \sigma_{R_{i}}\right) / \sigma_{R_{i}}$ and $F_{R_{i}}(x)=\phi((x-$ 
$\left.\left.E\left[R_{i}\right]\right) / \sigma_{R_{i}}\right)$. Substituting into (49) yields

$$
\begin{aligned}
E\left[\mu_{j}\right]= & \frac{E\left[R_{j}\right]}{N} \times\left(1-\left(\phi\left(-M_{j}\right)\right)^{N}\right) \\
& +\sigma_{R_{j}} \int_{-M_{j}}^{\infty} y \rho(y) \times \phi(y)^{N-1} d y .
\end{aligned}
$$

This completes the proof.

\section{REFERENCES}

[1] A. Sendonaris, E. Erkip, and B. Aazhang, "User cooperation diversity. part i. system description," IEEE Transactions on Communications, vol. 51, no. 11, pp. 1927-1938, November 2003.

[2] — , "User cooperation diversity. part ii. implementation aspects and performance analysis," IEEE Transactions on Communications, vol. 51, no. 11, pp. 1939-1948, November 2003.

[3] T. C.-Y. Ng and W. Yu, "Joint optimization of relay strategies and resource allocations in cooperative cellular networks," IEEE Journal on Selected Areas in Communications, vol. 25, no. 2, pp. 328-339, 2007.

[4] J. N. Laneman, D. N. C. Tse, and G. W. Wornell, "Cooperative diversity in wireless networks: Efficient protocols and outage behavior," IEEE Transactions on Information Theory, vol. 50, no. 12, pp. 3062-3080, 2004.

[5] E. Aktas, J. Evans, and S. Hanly, "Distributed decoding in a cellular multiple-access channel," IEEE Transactions on Wireless Communications, vol. 7, no. 1, pp. 241-250, 2008.

[6] A. Bletsas, A. Khisti, D. P. Reed, and A. Lippman, "A simple cooperative diversity method based on network path selection," IEEE Journal on Selected Areas in Communications, vol. 24, no. 3, pp. 659-672, March 2006.

[7] Y. Sung, S. Misra, L. Tong, and A. Ephremides, "Cooperative routing for distributed detection in large sensor networks," IEEE Journal on Selected Areas in Communications, vol. 25, no. 2, pp. 471-483, 2007.

[8] J. Huang, Z. Han, M. Chiang, and H. V. Poor, "Auction-based resource allocation for cooperative communications," IEEE Journal on Selected Areas in Communications, vol. 26, no. 7, pp. 1226-1237, 2008.

[9] O. Simeone, O. Somekh, G. Kramer, H. V. Poor, and S. Shamai, "Throughput of cellular systems with conferencing mobiles and cooperative base stations," EURASIP Journal on Wireless Communications and Networking, vol. 2008, pp. 1-14, 2008.

[10] W. Chen, L. Dai, K. Ben Letaief, and Z. Cao, "A unified crosslayer framework for resource allocation in cooperative networks," IEEE Transactions on Wireless Communications, vol. 7, no. 8, pp. 3000-3012, 2008.

[11] A. Papadogiannis, E. Hardouin, and D. Gesbert, "Decentralising multicell cooperative processing on the downlink: A novel robust framework," EURASIP Journal on Wireless Communications and Networking, August 2009.

[12] M. Dohler, A. Gkelias, and H. Aghvami, "2-hop distributed MIMO communication system," Electronics Letters, vol. 39, no. 18, pp. 1350 1351, 2003.

[13] A. D. Coso, U. Spagnolini, and C. Ibars, "Cooperative distributed MIMO channels in wireless sensor networks," IEEE Journal on Selected Areas in Communications, vol. 25, no. 2, pp. 402-414, 2007.

[14] Y. Rong and Y. Hua, "Optimal power schedule for distributed MIMO links," IEEE Transactions on Wireless Communications, vol. 7, no. 8, pp. 2896-2900, 2008.

[15] R. Mudumbai, D. R. Brown, U. Madhow, and H. V. Poor, "Distributed transmit beamforming: challenges and recent progress," IEEE Cоттиnications Magazine, vol. 47, no. 2, pp. 102-110, 2009.

[16] F. Kelly, "Charging and rate control for elastic traffic," European Transactions on Telecommunications, vol. 8, pp. 33-37, February 1997.

[17] H. J. Kushner and P. A. Whiting, "Convergence of proportional-fair sharing algorithms under general conditions," IEEE Transactions on Wireless Communications, vol. 3, no. 4, pp. 1250-1259, 2004.

[18] S. Borst, "User-level performance of channel-aware scheduling algorithms in wireless data networks," IEEE/ACM Transactions on Networking, vol. 13, no. 3, pp. 636-647, June 2005.

[19] T. Bu, L. Li, and R. Ramjee, "Generalized proportional fair scheduling in third generation wireless data networks," in Proc. INFOCOM 2006. The 25th Joint Conference of the IEEE Computer and Communications Societies. IEEE, April 2006, pp. 1-12.
[20] G. Caire, R. R. Muller, and R. Knopp, "Hard fairness versus proportional fairness in wireless communications: The single-cell case," IEEE Transactions on Information Theory, vol. 53, no. 4, pp. 1366-1385, April 2007.

[21] L. Li, M. Pal, and Y. R. Yang, "Proportional fairness in multi-rate wireless lans," in Proc. INFOCOM 2008. The 27th Joint Conference of the IEEE Computer and Communications Societies. IEEE, April 2008, pp. 1004-1012.

[22] G. Bianchi and A. T. Campbell, "A programmable mac framework for utility-based adaptive quality of service support," IEEE Journal on Selected Areas in Communications, vol. 18, no. 2, pp. 244-255, 2000.

[23] L. Shi, C. Liu, and B. Liu, "Network utility maximization for triple-play services," Computer Communications, vol. 31, no. 10, pp. 2257-2269, 2008.

[24] D. Xu, Y. Li, M. Chiang, and A. Calderbank, "Elastic service availability: utility framework and optimal provisioning," IEEE Journal on Selected Areas in Communications, vol. 26, no. 6, pp. 55-65, 2008.

[25] X. Zhang, M. Tao, and C. Ng, "Utility-based wireless resource allocation for variable rate transmission," IEEE Transactions on Wireless Communications, vol. 7, no. 9, pp. 3292-3296, 2008.

[26] Q. Gao, J. Zhang, and S. Hanly, "Cross-layer rate control in wireless networks with lossy links: Leaky-pipe flow, effective network utility maximization and hop-by-hop algorithms," IEEE Transactions on Wireless Communications, vol. 8, no. 6, pp. 3068-3076, 2009.

[27] A. Jalali, R. Padovani, and R. Pankaj, "Data throughput of CDMA-HDR a high efficiency-high data rate personal communication wireless system," in Proc. IEEE 51st VTC 2000-Spring Tokyo Vehicular Technology, vol. 3, January 2000, pp. 1854-1858.

[28] H. Zhou, P. Fan, and J. Li, "Cooperative proportional fairness scheduling for wireless transmissions," in IWCMC '09: Proceedings of the 2009 International Conference on Wireless Communications and Mobile Computing. New York, NY, USA: ACM, 2009, pp. 12-16.

[29] J. D. Parsons and A. S. Bajwa, "Wideband characterisation of fading mobile radio channels," IEE Proceedings F Communications, Radar and Signal Processing, vol. 129, no. 2, 1982.

[30] P. J. Smith, S. Roy, and M. Shafi, "Capacity of MIMO systems with semicorrelated flat fading," IEEE Transactions on Information Theory, vol. 49, no. 10, pp. 2781-2788, October 2003.

[31] M. R. McKay, P. J. Smith, H. A. Suraweera, and I. B. Collings, "On the mutual information distribution of OFDM-based spatial multiplexing: Exact variance and outage approximation," IEEE Transactions on Information Theory, vol. 54, no. 7, pp. 3260-3278, July 2008.

[32] E. Liu and K. K. Leung, "Proportional fair scheduling: Analytical insight under Rayleigh fading environment," in Proc. IEEE Wireless Communications and Networking Conference WCNC 2008, March/April 2008, pp. 1883-1888.

[33] R. Jain, The Art of Computer Systems Performance Analysis: Techniques for Experimental Design, Measurement, Simulation, and Modeling. New York: Wiley, April 1991.

[34] I. E. Telatar, "Capacity of multi-antenna gaussian channels," European Transactions on Telecommunications, vol. 10, pp. 585-595, November/December 1999.

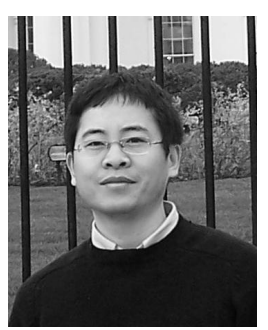

Erwu Liu received his M.S. and Ph.D degrees from the Department of Opto-Electronic Engineering, Huazhong University of Science \& Technology, CHINA, in 1998 and 2001, respectively.

He started his career at Alcatel-Lucent as a project manager in 2001, and then a senior research scientist at the Research \& Innovation (R\&I) Center there. In 2007, he left Alcatel-Lucent and joined Imperial College London as a researcher, working on wireless networks.

Currently, his research interests include: stochastic geometry, cooperative and cognitive networks, scheduling and opportunistic resource allocation, network utility maximization, and cross-Layer optimization, etc.

Dr. Liu is a member of the Alcatel-Lucent Technical Academy (ALTA), the ACM and the IEEE. 


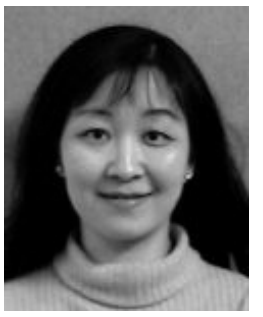

Qinqing Zhang received her Ph.D. degrees in electrical engineering from University of Pennsylvania, Philadelphia, PA, USA. She joined the Milton Eisenhower Research Center at the Johns Hopkins University Applied Physics Laboratory (JHU/APL) in May 2007. Prior to that, she was with Bell Labs, Alcatel-Lucent Technologies in New Jersey from 1998 to 2007.

Dr. Zhang is the recipient of numerous awards and scholarships, including the Bell Labs Presidents Gold Award in 2002. She is a senior member of IEEE. She serves on the editorial board of IEEE Transactions on Wireless Communications. She was the co-chair of workshop on Cooperative Communications and Networking - Theory, Practice and Applications for ICC2008, and the track organizer and chair in Milcom2007, Milcom2008, Milcom2009, and Milcom2010. She has been serving in technical program committees of various IEEE conferences, including IEEE Infocom, Globecom, ICC, WCNC, VTC, MWC, ICCNC, etc.

Her current research interests are mobile ad-hoc networks, cooperative communications and networks, and underwater acoustic communication systems and networks.

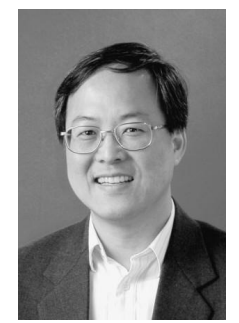

Kin K. Leung received his B.S. degree from the Chinese University of Hong Kong in 1980, and his M.S. and Ph.D. degrees in computer science from University of California, Los Angeles, in 1982 and 1985, respectively. He joined AT\&T Bell Labs in 1986 and worked at its successor companies, AT\&T Labs and Bell Labs of Lucent Technologies, until 2004. Since then, he has been the Tanaka Chair Professor in Internet Technology at Imperial College in London. He serves as the Head of Communications and Signal Processing Group and as the Deputy Director for the University Defense Research Center in Signal Processing in the Electrical Engineering Department at Imperial College. His research interests include networking, protocols, optimization and modeling issues for wireless broadband, sensor and ad-hoc networks.

He received the Distinguished Member of Technical Staff Award from AT\&T Bell Labs in 1994, and was a co-recipient of the 1997 Lanchester Prize Honorable Mention Award. He was elected as an IEEE Fellow in 2001. He receives the Royal Society Wolfson Research Merits Award from 2004 to 2009 . He has actively served on many conference committees. He is a member of the IEEE Fellow Evaluation Committee for Communications Society (2009 to 2011). He was a guest editor for the IEEE JOURNAL ON SELECTED AREAS IN COMMUNICATIONS (JSAC), IEEE WIRELESS COMMUNICATIONS and the MONET journal, and as an editor for the JSAC: Wireless Series and IEEE TRANSACTIONS ON WIRELESS COMMUNICATIONS. Currently, he is an editor for the IEEE TRANSACTIONS ON COMMUNICATIONS,INTERNATIONAL JOURNAL ON SENSOR NETWORKS and ACM COMPUTING SURVEY. 